\title{
DESENVOLVIMENTO DE CADEIRA DE RODAS HOSPITALAR
}

\author{
Júlio C. Augusto da Silva \\ Instituto Nacional de Tecnologia \\ Julio.silva@int.gov.br \\ Mauro Ricardo Rodrigues Melo \\ Instituto Nacional de Tecnologia \\ Mauro.melo@int.gov.br \\ Angelina Chacur Lopez \\ Instituto Nacional de Tecnologia \\ Angelina.lopez@int.gov.br \\ Thiago T. Muniz \\ Instituto Nacional de Tecnologia \\ Thiago.muniz@int.gov.br \\ Alvaro de Almeida Guimarães \\ Instituto Nacional de Tecnologia \\ alvaro.guimaraes@int.gov.br
}

Resumo: As cadeiras de rodas usualmente empregadas em hospitais são modelos domésticos ou adaptações destes, inadequadas para as necessidades do ambiente hospitalar.

Este artigo apresenta o resultado de um projeto de P\&D de uma cadeira de rodas projetada especificamente para o uso hospitalar.

$\mathrm{Na}$ fase de pesquisa foram levantados dados para apurar as características que uma cadeira de rodas hospitalar deve ter para atender as necessidades de segurança, conforto, funcionalidade, durabilidade, estética, manutenção e custos deste uso.

$\mathrm{Na}$ etapa de desenvolvimento, foi projetado um modelo de cadeira que considerasse esses parâmetros, de forma a atender com mais eficiência os usuários envolvidos com este produto: os pacientes e os profissionais de saúde.

O modelo proposto foi validado através de avaliações comparativas com os modelos atualmente empregados. As inovações propostas deram origem a pedidos de registro no INPI. 
Palavras-chave: tecnologia assistiva, equipamento médico hospitalar, cadeira de rodas, cadeira hospitalar, transferência de pacientes.

abstract: Wheelchairs used in hospitals are usually domestic models, inadequate for hospital needs.

This article presents the results of an $R \& D$ project of a wheelchair specifically designed to be used in hospitals.

During the researches, data were collected to determine the characteristics that a hospital wheelchair should have to meet the needs of safety, comfort, functionality, durability, aesthetics, and maintenance.

In the development stage, the new wheelchair was designed taking those parameters in consideration, in order to better meet its users: patients and health professionals.

The design was benchmarked with the existing models and validated. The proposed innovations gave rise to requests for registration in INPI.

Keywords : assistive technology, hospital medical equipment, wheelchair, hospital chair, patient transfers.

\section{INTRODUÇÃO}

O que se observa na maioria dos hospitais, especialmente os públicos, é o emprego de cadeiras que não foram projetadas para uso hospitalar, provocando diversos transtornos nos usuários, ou seja, nos pacientes e nos profissionais de saúde encarregados de auxiliar sua movimentação no hospital.

Neste projeto foi conduzida uma pesquisa em campo para levantar os parâmetros que uma cadeira deve ter para ser adequada às tarefas que devem cumprir no ambiente hospitalar. A seguir, um novo modelo foi desenvolvido, e as soluções propostas foram patenteadas.

O trabalho foi desenvolvido ao longo do ano de 2013 e contou com apoio financeiro da FAPERJ.

Uma cadeira de rodas planejada para as tarefas e necessidades do uso hospitalar facilitará o trabalho dos profissionais de saúde e reduzirá riscos e desconforto para os pacientes. Desta forma, contribuirá para humanização do ambiente hospitalar.

\section{DESENVOLVIMENTO}

Nos itens a seguir são apresentados o problema, objetivo do trabalho, método empregado para a pesquisa, o resultado da pesquisa e as soluções propostas.

\subsection{Problema}

$\mathrm{Na}$ internação hospitalar há uma série de atividades que não podem ser executadas no leito, tais como determinados exames, procedimentos médicos, higiene, fisioterapia, atividades físicas e recreação. Nesses momentos é necessário o 
translado dos pacientes dos seus quartos para outros ambientes. Para esses deslocamentos costumam ser empregadas cadeiras de rodas, salvo nos raros casos de pacientes em boas condições, que podem se deslocar pelo hospital caminhando, ou pacientes excessivamente debilitados, que necessitam de maca.

A cadeira de rodas empregada em hospitais tem uso coletivo, e as tarefas e necessidades da cadeira hospitalar difere muito das observadas nas cadeiras domésticas. No entanto, o que se observa na grande maioria dos hospitais é o emprego de cadeiras de rodas de uso doméstico, ou adaptações destas, não adequadas, portanto, à realidade hospitalar.

Como conseqüência dessa inadequação, observa-se diversos transtornos, tais como: risco de quedas, desconforto e constrangimentos para o paciente; esforços exagerados e lesões para os profissionais de saúde; baixa durabilidade e aumento de custos para os hospitais; falhas na assepsia.

\subsection{Objetivo}

O objetivo deste trabalho foi contribuir para melhoria da qualidade de vida dos pacientes durante a internação, melhora das condições de trabalho das equipes de saúde, e redução dos custos para os hospitais.

A melhora na qualidade de vida do paciente ocorrerá pelo aumento do conforto e segurança proporcionada por uma cadeira pensada para o translado de pessoas debilitadas, com soluções e componentes que aumentem a segurança nestes casos específicos. As condições de atendimento serão aprimoradas também pela adoção de medidas projetuais que permitam higienização mais rigorosa, tendo em vista a presença eventual de fluidos corporais.

A melhora nas condições de trabalho dos enfermeiros será proporcionada pela adoção de soluções que levem em conta as tarefas realizadas para movimentação do paciente.

A redução de custos será alcançada por cadeira pensada para o uso coletivo e intenso, que tenha manutenção simplificada, com componentes facilmente intercambiáveis. Indiretamente a redução de custos com atendimento hospitalar ocorrerá também pelo aumento da produtividade dos enfermeiros e maqueiros.

\subsection{Método de pesquisa aplicado}

A primeira etapa do trabalho foi o levantamento de dados para compreensão do problema e identificação das características que o produto deve ter para melhor atender às populações envolvidas. O levantamento foi realizado em três frentes: pesquisa bibliográfica, levantamento do estado da arte e pesquisa em campo.

A pesquisa em campo foi realizada junto à profissionais do ambiente hospitalar de alguma forma envolvidos com a cadeira de rodas. Foram abordados enfermeiros, maqueiros, diretores, chefe de clínicas, fisioterapeutas, encarregado de compras e técnicos de manutenção.

Dentre os instrumentos de pesquisa possíveis, optou-se pela entrevista, dado a flexibilidade que esta permite pela interação direta entre pesquisador e entrevistado. O modelo de entrevista escolhido foi o Semi-Estruturado, caracterizado por uma série 
de perguntas abertas feitas verbalmente em ordem prevista, mas na qual o entrevistador pode intervir ao longo da aplicação realizando alterações de última hora. LAVILLE (1999) e GIL (1999) apontam os aspectos positivos deste modelo de entrevista, que permite que o entrevistador acrescente perguntas, altere a ordem ou mesmo reformule as questões durante o contato com o entrevistado, de forma a melhor explorar seus conhecimentos e informações que pode fornecer.

O objetivo da pesquisa em campo foi conhecer a realidade das tarefas de translado de pacientes em ambiente hospitalar e aspectos técnicos da cadeira. A pesquisa foi realizada em cinco visitas técnicas a hospitais públicos e privados no Rio de Janeiro, entre janeiro e maio de 2013.

Durante a pesquisa foram analisados os procedimentos relacionados ao translado dos pacientes. Foram levantadas como é realizado o translado, quem são os responsáveis, quais técnicas de transferência $^{1}$ são empregadas, existência de equipamentos para auxiliar a transferência, riscos e formas de mitigá-los etc.

Já sobre a manutenção, foram levantados dados sobre a durabilidade das cadeiras, quais componentes apresentam maior índice de quebras, onde e como é realizada a manutenção preventiva e corretiva.

Também foram observadas características dos ambientes, como largura de corredores, portas, espaço disponível para armazenamento, áreas de manobra, condições do piso, rampas, existência de barreiras arquitetônicas à movimentação, etc.

\subsection{Resultados}

O principal problema do translado de pacientes em hospitais, segundo a pesquisa realizada, é a transferência de pacientes que não conseguem ficar de pé. Este resultado também foi apontado nas pesquisas de ROSSI (2001), GALLASCH (2003), ALEXANDRE (2000) e COMÉLIO (2005). Os enfermeiros realizam a transferência erguendo os pacientes pelas axilas e pernas (figura 1), técnica também descrita em ALEXANDRE (op. Cit.). Este procedimento, embora seja o mais adequado quando não há elevadores para a transferência do paciente, não é isento de limitações. Mesmo quando bem executado, o procedimento pode provocar dores, constrangimentos e riscos de quedas, especialmente em pacientes idosos, recém operados ou doloridos. Os enfermeiros também sofrem com essa manobra. Como muitos dos pacientes têm sobrepeso, este procedimento, mesmo quando executado com a técnica correta, representa esforço excessivo, risco para a coluna e articulações do profissional de saúde, sendo o principal responsável por doenças ocupacionais nesta classe de trabalhadores, segundo ROSSI (op. Cit.) e COMÉLIO (op. Cit.).

\footnotetext{
1 Neste artigo o termo "transferência" será empregado para designar a operação de passagem do paciente do leito para a cadeira de rodas e vice versa, onde o profissional de saúde deve amparar ou carregar o paciente. Para a movimentação do paciente entre os ambientes hospitalares, já sentado na cadeira de rodas, será empregado o termo "translado".
} 


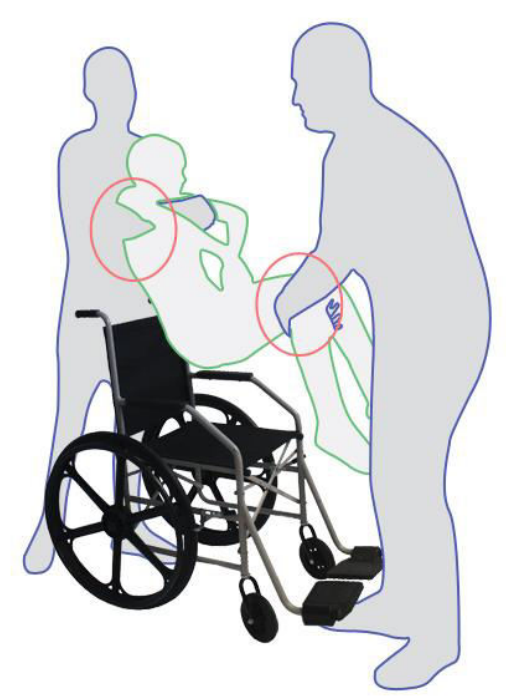

Figura 1 - Procedimento para transferência de pacientes debilitados da cadeira para a cama (figura elaborada pelo autor, com base na pesquisa realizada).

Além do processo de transferência dos pacientes, outra fonte de dores e doenças ocupacionais para os profissionais de saúde são cadeiras de rodas com elementos mal dimensionados e posicionados, (figura 2). A situação se agrava quando é preciso conduzir cadeiras em aclives. O problema também foi apontada por GALLASCH (2003) e COMÉLIO (2005).

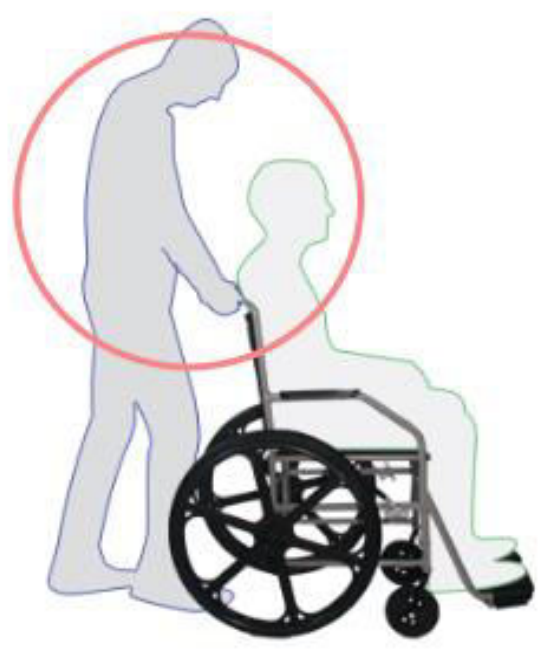

Figura 2 - manípulos mal localizados forçam uma postura errada da coluna e torção no pulso (figura elaborada pelo autor, com base na pesquisa realizada).

Para o paciente, a ergonomia das cadeiras de rodas empregadas em hospitais também se mostra inadequada e fonte de riscos. Em ambiente hospitalar ocorre por vezes a necessidade de transladar pacientes debilitados ou com dificuldade de manter a postura sentada. A movimentação destes pacientes em cadeira de rodas apresenta 0 risco de quedas, especialmente quando a cadeira passa por um declive ou degrau, pois o paciente pode tombar para frente. 
Outro problema ergonômico observado na pesquisa foi o desconforto provocado por falta de regulagens de rápida operação. As pernas de alguns usuários, por exemplo, não se apóiam adequadamente no apoio do pé, ficando penduradas (figura 3) ou muito dobradas.

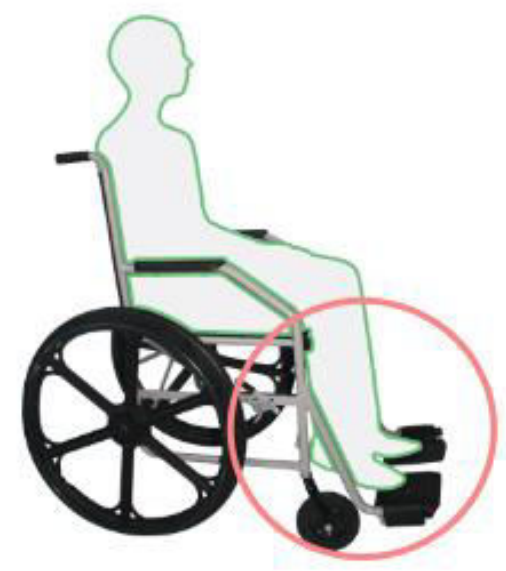

Figura 3 - paciente conduzido com as pernas penduradas, sem tocar no apoio do pé (figura elaborada pelo autor, com base na pesquisa realizada).

Além dos problemas ergonômicos e antropométricos, outro transtorno que as cadeiras provocam no ambiente hospitalar é o espaço ocupado na recepção e corredores, freqüentemente superlotados, onde várias unidades precisam ficar estacionadas à disposição dos usuários.

Muitas das cadeiras empregadas em hospitais tem uma solução de compactação para reduzir o espaço ocupado quando estacionadas. O sistema de empregado normalmente é pantográfico, onde uma articulação na estrutura aproxima as duas laterais da cadeira reduzindo pela metade o espaço ocupado. Este sistema é amplamente empregado em cadeiras de rodas domésticas, mas apresenta inconvenientes quando aplicado em cadeiras destinadas ao ambiente hospitalar. Esta solução fragiliza a estrutura da cadeira, o que provoca freqüentes quebras devido ao uso coletivo e intenso no hospital (figura 4). Desta forma, a durabilidade das cadeiras fica muito reduzida. Outro inconveniente da solução pantográfica, quando empregado em cadeiras de uso hospitalar, é facilitar o furto. Como a cadeira dobrada cabe em porta-malas de automóveis, é comum o furto quando o paciente recebe alta e é conduzido, pelo acompanhante, até o seu veículo ou taxi. Por fim, a solução pantográfica tende a aumentar o peso e os custos das cadeiras. 


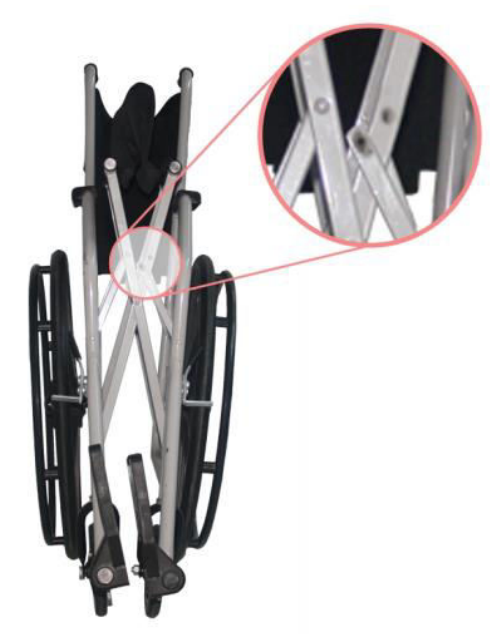

Figura 4 - o sistema pantográfico é frágil, sujeito à quebras na articulação (figura elaborada pelo autor, com base na pesquisa realizada).

Outra dificuldade comum no translado de pacientes em cadeiras em ambiente hospitalar é a manobra em corredores obstruídos, elevadores lotados, recepções congestionadas.

As cadeiras de rodas necessitam de um espaço grande para fazer curvas (figura 5 , , o que nem sempre se encontra em ambientes apertados ou congestionados. Esta limitação ocorre porque as cadeiras possuem rodas grandes e fixas atrás, e rodas pivotantes apenas na frente.

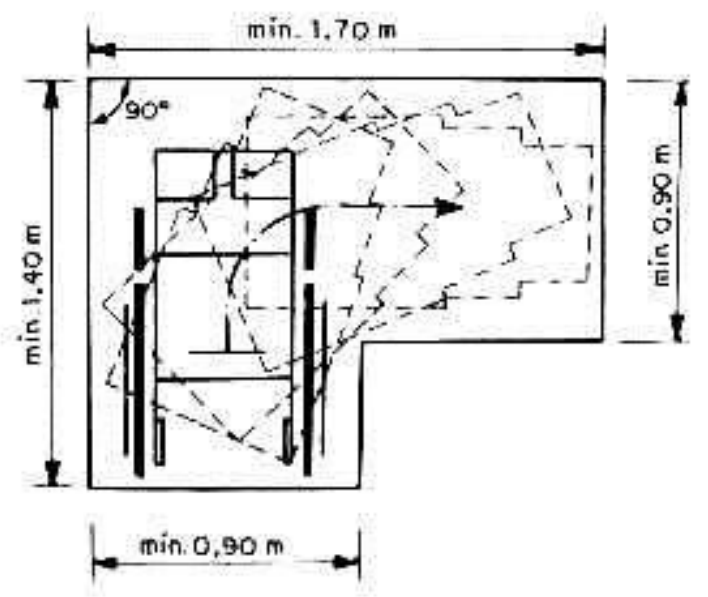

Figura 5 - área mínima de manobra de cadeira tradicional (figura baseada em ABNT 2004)

Por fim, outra característica que apontada na pesquisa como mal resolvida é a aparência. A cadeira de rodas tradicional é considerada um dos componentes que concorre para o aspecto negativo que se observa neste ambiente. Geralmente há excesso de metal aparente, uso intensivo de aço inoxidável, formas retas, cores cromadas ou pretas. Esses elementos costumam estar associados ao ambiente cirúrgico, odontológico ou ainda à dramas como doenças ou acidentes. 


\subsection{Propostas}

Para minimizar os riscos e desconfortos do procedimento de transferência de pacientes debilitados foi desenvolvido um sistema de transferência, composto por um assento e encosto que se destacam da estrutura da cadeira, se comportando como uma maca (figura 6).

Com essa solução simples evita-se o contato direto no corpo do paciente, preservando sua intimidade física. O peso fica distribuído por todo o corpo, não concentrado nas axilas, reduzindo o desconforto e dores.

Esta solução também reduz risco de lesões por esforço excessivo nos profissionais de saúde, pois as alças estão posicionadas de forma a permitir uma postura mais adequada durante o procedimento. As alças também oferecem mais firmeza para movimentação, e permitem a participação de até quatro profissionais, caso o paciente seja muito pesado.
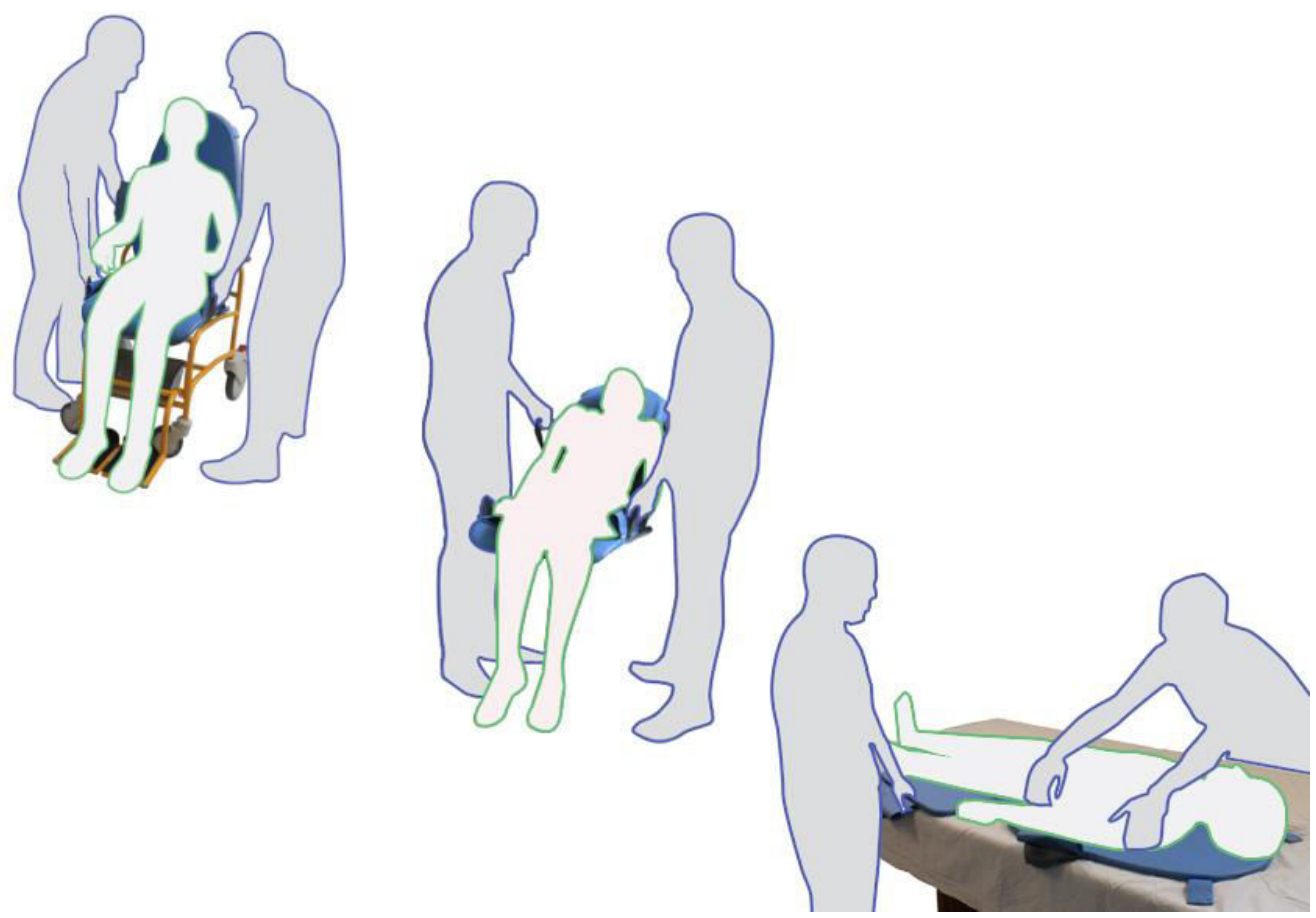

Figura 6 - sistema de transferência proposto: o assento e encosto da cadeira se destacam da estrutura (figura elaborada pelo autor, com base na pesquisa realizada).

Para minimizar o risco de queda quando do translado de pacientes debilitados, na cadeira proposta o encosto é mais alto, apoiando o paciente até a cabeça. A inclinação do encosto também foi aumentada, para jogar o centro de gravidade para trás e aumentar a estabilidade. Essas soluções aumentam a segurança de pacientes debilitados, reduzindo o risco de tombamento para frente, além de proporcionar mais conforto.

Já o problema da regulagem do apoio do pé, foi resolvido com um sistema de regulagem da altura do apoio do pé com engates rápidos, de fácil ajuste (figura 7). 

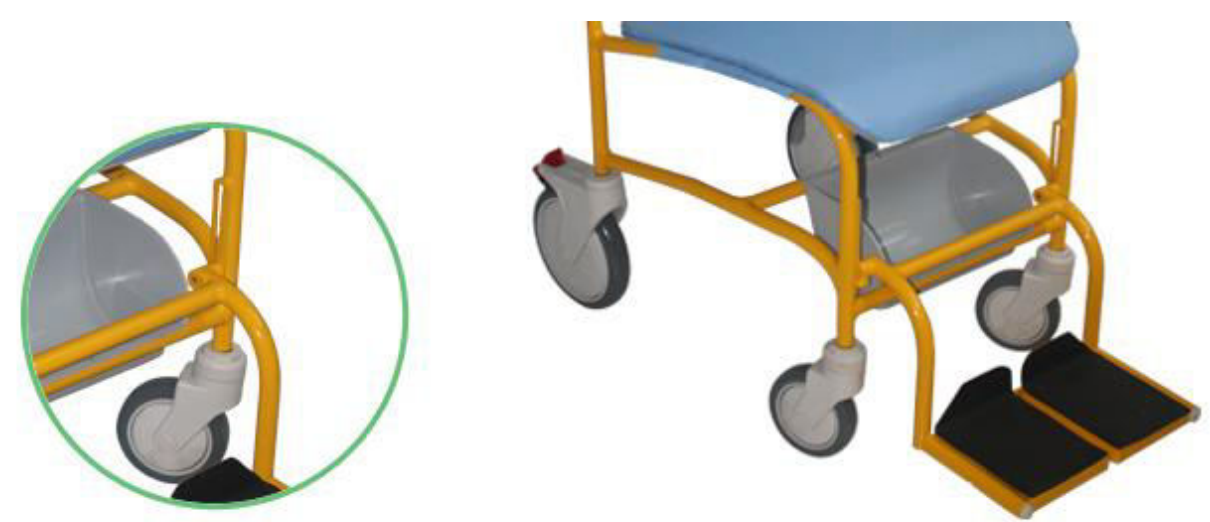

Figura 7 - apoio do pé regulável, com sistema de engate rápido (figura elaborada pelo autor, com base na pesquisa realizada).

As condições de trabalho do profissional de saúde foram melhoradas também com intervenções nos controles da cadeira. Os manípulos de condução foram posicionados mais alto e com uma inclinação em relação à horizontal (figura 8), seguindo dados antropométricos disponíveis em INT (1998), DIFRENT (1983) e DREYFUSS (2010). Com isso foi possível reduzir o esforço físico, principalmente na subida de rampas, e o desconforto na coluna e articulação do pulso do condutor. Já os freios são acionados por pedal, diretamente nas rodas.

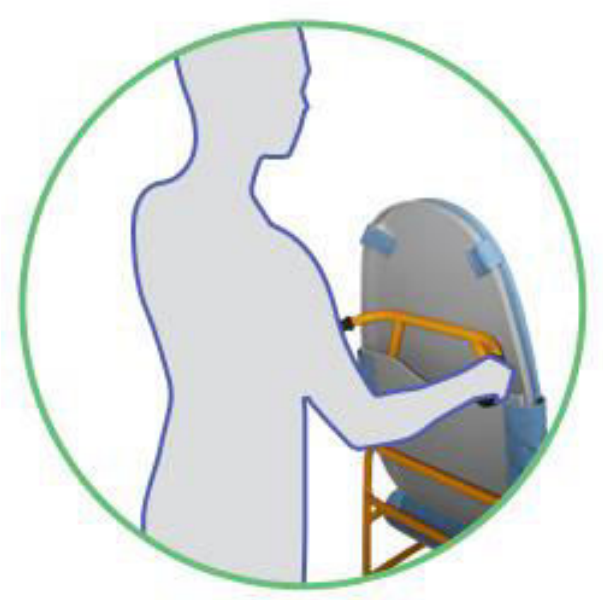

Figura 8 - manípulos mais altos e inclinados para manter o pulso na posição reta (figura elaborada pelo autor, com base na pesquisa realizada).

A proposta para reduzir o espaço ocupado pelas cadeiras estacionadas, sem fragilizar o produto, é um sistema de compactação baseado na geometria da estrutura. No sistema proposto, a estrutura da cadeira tem uma forma levemente cônica, permitindo que uma se acople em outra (figura 9) sem uso de articulações. 

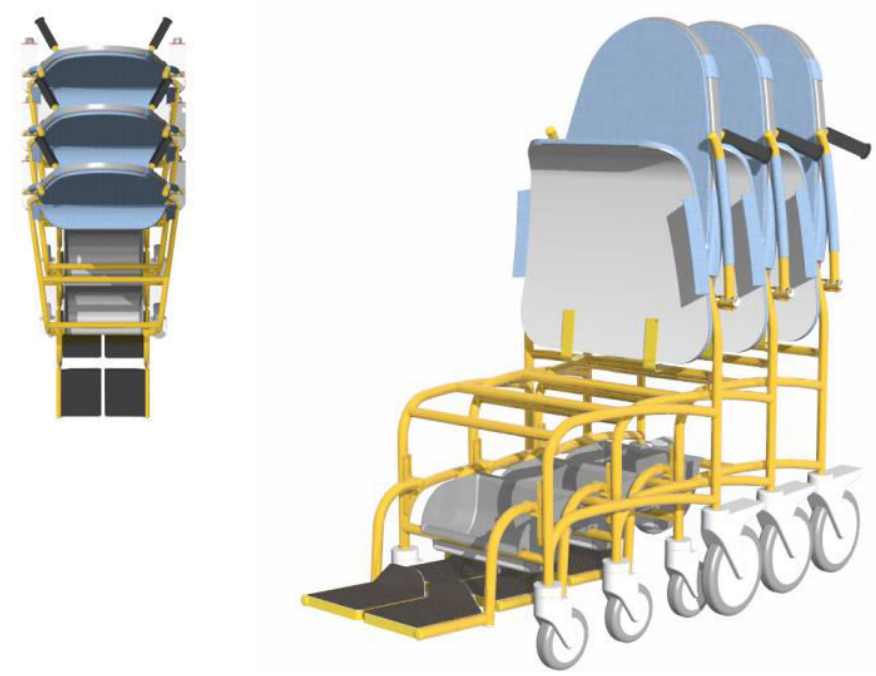

Figura 9 - estrutura com geometria cônica permite o acoplamento de várias cadeiras (figura elaborada pelo autor, com base na pesquisa realizada).

O sistema proposto poupa mais espaço do que o sistema pantográfico quando há várias unidades, situação comum em hospitais. Além deste ganho, o sistema proposto não emprega articulações nem partes móveis na estrutura. Com isso a estrutura é mais simples, durável, leve e menos dispendiosa. Outro benefício desta solução é dificultar o furto de cadeiras, pois o modelo não cabe em porta-malas.

A inovação proposta para melhorar a capacidade de manobra das cadeiras é a adoção de quatro rodas pivotantes. Esta solução proporciona considerável ganho na capacidade de manobra, reduzindo a área necessária para curvas. Esta proposta permite também deslocamentos na diagonal ou na lateral, facilitando muito a manobra em espaços contidos.

Para minimizar a aparência dramática normalmente associada às cadeiras de rodas, no modelo proposto buscou-se soluções estéticas que remetessem à otimismo, esperança, acolhimento e amparo, com adoção de cores alegres, formas delgadas, linhas arredondadas. Foram propostas diversas combinações de cores para estrutura, estofamentos e rodas. A variedade de combinação de cores também irá contribuir para quebrar a monotonia do ambiente onde tradicionalmente predominam cadeiras pretas. Além disso, a variação de cores permitirá fazer diferenciação de setores hospitalares.

\section{CONCLUSÃO}

O produto foi validado em testes que simularam as diversas situações de uso, como transferência de pacientes entre cadeira e cama e vice versa, subida e descida de rampas, condução da cadeira por espaços limitados. Para o teste foram empregados voluntários representando os pacientes e profissionais de saúde, selecionados entre diferentes percentis antropométricos. Os testes foram realizados compearanto a cadeira proposta com os modelos tradicionais.. 
Como resultado, foi confirmado que o desempenho da cadeira proposta superou o registrado nas cadeiras tradicionais para as tarefas exigidas no ambiente hospitalar.

O produto foi registrado com quatro pedidos no INPI, sendo uma Patente de Inovação e três Modelos de Utilidade. Ao final do registro o produto será repassado ao setor produtivo para comercialização.

Dado as limitações de um projeto desta natureza, a presente pesquisa não se aprofundou em necessidades especificas de determinados setores hospitalares, como ortopedia, pediatria, cardiologia e queimados. Também não abordou necessidades sanitárias e banho, mas tão somente as necessidades relacionadas à transferência e translado. Os demais temas, igualmente mal resolvidos nas cadeiras empregadas atualmente, são, portanto, bons objetos para futuras pesquisas.

\section{REFERÊNCIAS}

ALEXANDRE, Neusa Maria; ROGANTE, Maria Marilene. Movimentação e transferência de pacientes: aspectos posturais e ergonômicos. In Revista da Escola de enfermagem da USP. 2000 jun, V. 35, n 2, p. 165,73.

Associação Brasileira de Normas Técnicas. NBR ISO 7176-1 Cadeira de rodas Parte 1: Determinação de estabilidade estática. Rio de Janeiro, 2009

Associação Brasileira de Normas Técnicas. NBR ISO 7176-7 Cadeira de rodas Parte 7: Medição de dimensões de assentos e rodas. Rio de Janeiro, 2009

Associação Brasileira de Normas Técnicas. NBR 9050 Acessibilidade a edificações, mobiliário, espaços e equipamentos urbanos. Rio de Janeiro, 2004

COMÉLIO, M. E.; ALEXANDRE, N. M. C. Avaliação de uma cadeira de banho utilizada em ambiente hospitalar: uma abordagem ergonômica. In Revista Pesquisa Brasileira de Enfermagem. 2005 jul-ago; 58(4):405-10.

DREYFUSS, Henry. As medidas do homem e da mulher, fatores humanos no design. São Paulo: Bookman, 2010.

DIFFRIENT, Niels. Human scale Manual. Massachusetts: MIT Press, 1983.

GALLASCH, Cristiane Helena; ALEXANDRE, Neusa Maria Costa. Avaliação dos riscos ergonômicos durante a movimentação e transporte de pacientes em diferentes unidades hospitalares. In Revista de Enfermagem da UERJ. 2003, 11:252-60. GIL, Antonio Carlos. Métodos e técnicas de pesquisa social. São Paulo: Atlas, 1999. 
IIDA, Itiro Ergonomia, projeto e produção. Rio de Janeiro: Edgar Blücher, 1993.

INT. ERGOKIT - Banco de Dados Antropométricos da População Brasileira. Rio de Janeiro: MCT, 1998.

INT, Manual de Aplicação dos Dados Antropométricos. Rio de Janeiro: MCT, 1986.

ISO 7176-8 Wheelchairs Part 8: Requirements and test methods for static, impact and fatigue strengths. 1998.

LAVILLE, Cristian, DIONE, Jean. A construção do Saber. Belo Horizonte: Ed. UFMG,1999.

RESENDE, Luiz Cláudio Cunha em "A Cor no Ambiente Hospitalar", in Anais do I Congresso Nacional da Abdeh - IV Seminário de Engenharia Clínica. 2004 ROSSI, C. G; ROCHA R. M; ALEXANDRE N. M.C. Aspectos ergonômicos na transferência de pacientes: um estudo realizado com trabalhadores de uma central de um hospital universitário. In Revista da Escola de Enfermagem da USP 2001; 35(3):249-56. 